\title{
LAGUERRE POLYNOMIALS, WEIGHTED DERANGEMENTS, AND POSITIVITY*
}

\author{
DOMINIQUE FOATA $\dagger$ AND DORON ZEILBERGER $\ddagger$
}

\begin{abstract}
A calculation of the linearization coefficients of the (generalized) Laguerre polynomials $L_{n}^{(\alpha)}(x)$ is proposed by means of analytic and combinatorial methods. This paper extends to the case of an arbitrary $\alpha$ a combinatoric and analytic result due to Askey, Ismail, and Koornwinder and Even and Gillis.
\end{abstract}

Key words. Laguerre polynomials, linearization coefficients, weighted derangements, MacMahon Master Theorem ( $\beta$-extension)

AMS(MOS) subject classifications. 33A65, 05A 15

1. Introduction. Let $\left(p_{n}(x)\right)$ be a sequence of polynomials, orthogonal with respect to a weight function $w$. One of the aspects of the linearization of the product of the $p_{n}(x)$ 's is the evaluation of integrals of the form

$$
\mathscr{I}=\int \prod_{i=1}^{m} n_{i} d w,
$$

for the classical polynomials, such as the Jacobi, Meixner, Charlier, Laguerre, and Hermite polynomials. What is meant by evaluation is either the determination of a formula for $\mathscr{I}$ in terms of the classical hypergeometric series (see, e.g., the fantastic formula found by Rahman [ $\mathrm{Ra}$ ] for the Jacobi polynomials involving the series ${ }_{9} F_{8}$ ), or the geometric interpretation of $\mathscr{I}$ as a generating polynomial for some combinatorial objects, such as permutations or partitions (see, e.g., the present paper for the Laguerre polynomials, or the article by Zeng [ Ze] for the Meixner, Krawtchouk, and Charlier polynomials). Of course, for many problems it is essential that $\mathscr{I}$ be positive. In Rahman's formula, for instance, all the hypergeometric series involved in the formula are positive-this is the easy part-the difficult part is the derivation of the formula itself. In the combinatorial approach the positivity of $\mathscr{I}$ also appears as a byproduct, the essential part being played by the construction of the geometric setup for the integral $\mathscr{I}$.

We want to illustrate this in this paper by making a systematic study of integrals of products of (general) Laguerre polynomials $L_{n}^{(\alpha)}(x)$. Recall that those polynomials are orthogonal with respect to the weight function $x^{\alpha} e^{-x}$ over $\mathbb{R}_{+}$. They may also be defined by their generating function

$$
\sum_{n=0}^{\infty} L_{n}^{(\alpha)}(x) u^{n}=(1-u)^{-\alpha-1} \exp \frac{-x u}{1-u} .
$$

Let $m$ be a positive integer, $\mathbf{n}$ be a sequence $\mathbf{n}=\left(n_{1}, \cdots, n_{m}\right)$ of nonnegative integers, $\Lambda$ be a sequence $\Lambda=\left(\lambda_{1}, \cdots, \lambda_{m}\right)$ of real numbers and let $A(\mathbf{n} ; \alpha), B(\mathbf{n} ; \Lambda ; \alpha)$ be the

* Received by the editors January 14, 1988; accepted for publication May 5, 1988.

† Département de mathématique, Université Louis-Pasteur, 7, rue René-Descartes, F-67084 Strasbourg, France. The work of this author was supported by the French Coordinated Research Program in Mathematics and Computer Science.

$¥$ Department of Mathematics, Drexel University, Philadelphia, Pennsylvania 19104. The work of this author was partially supported by the National Science Foundation. 
integrals

$$
\begin{gathered}
A(\mathbf{n} ; \alpha)=(-1)^{n_{1}+\cdots+n_{m}} \int_{0}^{\infty}\left(\prod_{i=1}^{m} L_{n_{i}}^{(\alpha)}(x)\right) x^{\alpha} e^{-x} d x ; \\
B(\mathbf{n} ; \Lambda ; \alpha)=\int_{0}^{\infty}\left(\prod_{i=1}^{m} L_{n_{i}}^{(\alpha)}\left(\lambda_{i} x\right)\right) x^{\alpha} e^{-x} d x .
\end{gathered}
$$

Also introduce the expressions:

$$
\mathscr{I}(\mathbf{n} ; \alpha)=\frac{1}{\Gamma(\alpha+1)}\left(\prod_{i=1}^{m} n_{i} !\right) A(\mathbf{n} ; \alpha)
$$

and

$$
J(\mathbf{n} ; \Lambda ; \alpha)=\frac{1}{\Gamma(\alpha+1)}\left(\prod_{i=1}^{m} n_{i} !\right) B(\mathbf{n} ; \Lambda ; \alpha) .
$$

The first goal of the present paper is to give a combinatorial interpretation to $\mathscr{I}(\mathbf{n} ; \alpha)$ and $J(\mathbf{n} ; \Lambda ; \alpha)$, that is, to show that those two expressions are generating polynomials for permutations by certain statistics. In particular, $\mathscr{I}(\mathbf{n} ; \alpha)$ will be shown to be the generating function for a special class of permutations, called $m$-derangements by the number of cycles.

To make it more precise let us now define those combinatorial objects. Let $A_{1}=$ $\left\{a_{1,1}, \cdots, a_{1, n_{1}}\right\}, \cdots, A_{m}=\left\{a_{m, 1}, \cdots, a_{m, n_{m}}\right\}$ be $m$ mutually disjoint finite sets. The group of the permutations of $A=A_{1}+\cdots+A_{m}$ is denoted by $\mathscr{P}(\mathbf{n})$. Consider any permutation belonging to $\mathscr{P}(\mathbf{n})$. An element $a_{i, j}$ of $A_{i}$ is said to be $(\pi)$-incestuous, if it is sent by $\pi$ to one of its own kind, i.e., if $\pi\left(a_{i, j}\right) \in A_{i}$. Denote by $\operatorname{Inc}_{i} \pi$ the set of the incestuous elements of $\pi$ in $A_{i}$ and let Inc $\pi=\sum_{i}$ Inc $_{i} \pi$. If a permutation $\pi$ has no incestuous element, (i.e., if $\pi\left(A_{i}\right) \cap A_{i}=\varnothing$ for all $i$ ), it is called an $m$-derangement. Denote by $\mathscr{D}(\mathbf{u})$ the subset of $\mathscr{P}(\mathbf{n})$ consisting of all the $m$-derangements. Finally, for each permutation $\pi$ in $\mathscr{P}(\mathbf{n})$ denote by cyc $\pi$ its number of cycles and define its $(w)$ weight by

$$
w(\pi)=(\alpha+1)^{\operatorname{cyc} \pi} .
$$

The first result is the following theorem.

THEOREM 1. For each variable $\alpha$ we have

$$
\mathscr{I}(\mathbf{n} ; \alpha)=\sum_{\pi} w(\pi)=\sum_{\pi}(\alpha+1)^{\mathrm{cyc} \pi} \quad(\pi \in \mathscr{D}(\mathbf{n})) .
$$

As for the combinatorial interpretation of $J(\mathbf{n}, \Lambda ; \alpha)$, introduce another $(W)$-weight as follows:

$$
W(\pi)=w(\pi) \prod_{i=1}^{m}\left(1-\lambda_{i}\right)^{\left|\operatorname{Inc}_{i} \pi\right|}\left(-\lambda_{i}\right)^{\left|A_{i} \backslash \operatorname{Inc}_{i} \pi\right|} .
$$

The corresponding result for $J(\mathbf{n} ; \Lambda ; \alpha)$ is then as follows in Theorem 2 .

THEOREM 2. For each variable $\alpha$ we have

$$
J(\mathbf{n} ; \Lambda ; \alpha)=\sum_{\pi} W(\pi) \quad(\pi \in \mathscr{P}(\mathbf{n})) .
$$

When $\Lambda=\mathbf{1}=(1,1, \cdots, 1)$, the $W$-weight reduces to

$$
W(\pi)= \begin{cases}(-1)^{n_{1}+\cdots+n_{m}} W(\pi) & \text { if } \pi \text { is an } m \text {-derangement, } \\ 0 & \text { otherwise. }\end{cases}
$$


Thus, Theorem 1 is just a particular case of Theorem 2 . Accordingly, only Theorem 2 will be proved in this paper.

The second goal of this paper is to study the positivity of $\mathscr{I}$ and $J$. For $\mathscr{I}$ it is easy; the positivity property of $\mathscr{I}(\mathbf{n} ; \alpha)$ for $\alpha>-1$ follows immediately from our combinatorial interpretation (1.6). As such, it is in no case a new result. It follows from more general theorems that cover the case of several classical polynomials, including the Laguerre polynomials (see, e.g., the basic paper by Askey [As1], or his monograph [As2]). The positivity property can also be proved by means of a simple analytic argument, as derived by Askey and [ As-Is], [ As-Is-Ko], [ As-Ga]. As they have noted, the generating function (1.1) yields the following identity:

$$
\sum_{\mathbf{n}} A(\mathbf{n} ; \alpha) x_{1}^{n_{1}} \cdots x_{m}^{n_{m}}=\frac{\Gamma(\alpha+1)}{\left(1-e_{2}-2 e_{3}-\cdots-(m-1) e_{m}\right)^{\alpha+1}},
$$

where $\mathbf{n}=\left(n_{1}, \cdots, n_{m}\right)$ runs over all sequences of $m$ nonnegative integers and where $e_{j}$ denotes the $j$ th elementary symmetric function in $x_{1}, \cdots, x_{m}$. The positivity of $\mathscr{I}(\mathbf{n} ; \alpha)$ for $\alpha>-1$ is then clear from (1.8).

As far as positivity is concerned, the combinatorial approach derived in this paper refines the analytic result in the following sense. Not only is $\mathscr{I}(\mathbf{n} ; \alpha)$ shown to be positive, but it is, in fact, a polynomial in $(\alpha+1)$ with positive integral coefficients.

When $\alpha=0$, the integrand in $\mathscr{I}(\mathbf{n} ; \alpha)$ is a product of simple Laguerre polynomials and Theorem 1 implies that $\mathscr{I}(\mathbf{n} ; 0)$ is equal to the number of $m$-derangements, a result due to Even and Gillis [ Ev-Gi]. Other proofs can be found in [ Ja] and [ Sa-Vi]. In [ AsIs, pp. 857-858] the authors were very close to finding a combinatorial interpretation of $\mathscr{I}(\mathbf{n} ; \alpha)$ for an arbitrary $\alpha$. What was missing in their derivation was an appropriate extension of the "Master Theorem" of MacMahon [ Mac, pp. 97-98]. It is also the purpose of this paper to state and prove such a theorem (see the $\beta$-extension of the Master Theorem in $\S 3$ ). As shown in $\S 5$, that $\beta$-extension, together with the calculations made by Askey and his coauthors, suffice to establish Theorem 2. We also give a truly combinatorial proof in $\S 4$, after having recorded the material on injection counting in $\S 2$.

The positivity of $J(\mathbf{n} ; \Lambda ; \alpha)$ for other values of $\Lambda$ is more difficult to handle. The combinatorial interpretation (1.7) brings no evidence of the positivity for an arbitrary $\alpha \geqq 0$. Koornwinder's inequality [Ko] says that when $m=3, \Lambda=(\lambda, 1-\lambda, 1)$, then

$$
\alpha \geqq 0, \quad 0 \leqq \lambda \leqq 1 \Rightarrow J(\mathbf{n}, \Lambda, \alpha) \geqq 0 .
$$

Also the strict inequality holds when $n_{1}=0$ and $0<\lambda<1$. Inequality (1.9) is certainly a deep result. To derive further extensions for $m>3$, Askey, Ismail, and Koornwinder [As-Is-Ko] have used the orthogonality property of the Laguerre polynomials and also the so-called "old expansion" of the same polynomials. As it is (too) easy to prove both the orthogonality relation and the old expansion by combinatorial methods, we shall not concern ourselves with the general extension. We shall concentrate on re-proving Koornwinder's inequality (Theorem 3 of $\S 6$ ). The argument developed is very similar to the one developed by Ismail and Tamhankar [ Is-Ta] or Gillis and Zeilberger [ Gi-Ze]. We may say that the proof of Theorem 3 is the rewriting of the latter authors' paper using the spirit and method of the former ones.

2. Cycles. We will need three results that are fundamental in the current combinatorial interpretation of special functions. First, the generating function for the set $\mathscr{S}_{n}$ of all the permutations on $n$ elements by number of cycles is given by (see, e.g., [ Ri, p. 78]):

$$
w\left(\mathscr{S}_{n}\right)=\sum_{\pi \in \mathscr{S}_{n}} w(\pi)=(\alpha+1)_{n}=(\alpha+1)(\alpha+2) \cdots(\alpha+n) .
$$


Let $1 \leqq k \leqq n$ and $S$ be a $(n-k)$-element subset of the $n$-element

$$
[n]=\{1 ; 2 ; \cdots, n\} \text {. }
$$

The set of injections from $S$ into [ $n]$ will be denoted by $\operatorname{Inj}(S, n)$. An injection from $S$ to $[n]$ consists of a (possibly empty) collection of cycles within $S$ and some simple paths that wander in $S$, but terminate at a point outside $S$. Similarly, denote by cyc $\pi$ the number of cycles of $\pi$ and define its weight by $w(\pi)=(\alpha+1)^{\text {cyc } \pi}$.

For example, if $S=\{1,2,3,4,5,6\}$ and $n=9$, then $(1,3),(2), 4 \rightarrow 5 \rightarrow 7$, $6 \rightarrow 8$ is an injection with weight $(\alpha+1)^{2}$.

The result analogous to (2.1) reads (see [ Fo-St, Lemma 2.1]) as follows: if card $S=n-k$, then

$$
w(\operatorname{Inj}(S, n))=\sum_{\pi} w(\pi)=(\alpha+1+k)_{n-k} \quad(\pi \in \operatorname{Inj}(S, n)) .
$$

The third result is concerned with the calculation of the generating function for a particular class of permutations of the set $A=A_{1}+\cdots+A_{m}$ (see the notation introduced in $\S 1)$. For each $i=1, \cdots, m$ let $T_{i}$ be a given subset of $A_{i}$ of cardinality $\left(n_{i}-k_{i}\right)$ and denote by $\mathscr{P}(\mathbf{T} \subset \mathrm{Inc})$ the set of all permutations $\pi$ of $A$ satisfying $T_{i} \subset \operatorname{Inc}_{i} \pi$ for all $i$.

LEMMA. We have

$$
w(\mathscr{P}(\mathbf{T} \subset \mathrm{Inc}))=(\alpha+1)_{k_{1}}+\cdots+k_{m} \prod_{i=1}^{m}\left(\alpha+1+k_{i}\right)_{n_{i}-k_{i}} .
$$

Proof. From (2.1) and (2.2) it follows that the right-hand side of (2.3) is the generating function for the product $\mathscr{P}(\mathbf{k}) \times \prod_{i=1}^{m} \operatorname{Inj}\left(T_{i}, n_{i}\right)$ by $w$. To prove the lemma it then suffices to construct a $w$-weight preserving bijection $\pi \rightarrow\left(\pi_{1}, \cdots, \pi_{m}, \sigma\right)$ of $\mathscr{P}(\mathbf{T} \subset \mathrm{Inc})$ onto that product. Write $\pi$ in cycle form. Then in each cycle of $\pi$ delete all the elements of $T=T_{1} \cup \cdots \cup T_{m}$. What remains is a permutation written in cycle form. Call it $\sigma$.

To get $\pi_{i}$ take all the cycles of $\pi$ consisting only of elements of $T_{i}$. Also take the connected portions of $T_{i}$ lying in other cycles. Doing this will result in a certain number of paths that wander through $T_{i}$ but terminate in an element not in $T_{i}$.

Clearly, $\sigma$ belongs to $\mathscr{P}(\mathbf{k})$ and each $\pi_{i}$ is an injection of $S_{i}$ into $A_{i}$. Moreover, the total number of cycles of $\sigma, \pi_{1}, \cdots, \pi_{m}$ is equal to cyc $\pi$. Thus, the mapping is $w$-preserving. The reverse construction is immediate.

Example. Take the following:

$$
\begin{array}{lll}
n_{1}=6, & n_{2}=6, & n_{3}=6, \\
k_{1}=3, & k_{2}=3, & k_{3}=3, \\
T_{1}=\left\{a_{1}, a_{2}, a_{3}\right\}, & T_{2}=\left\{b_{1}, b_{2}, b_{3}\right\}, & T_{3}=\left\{c_{1}, c_{2}, c_{3}\right\}, \\
\pi=\left(a_{1} a_{2}\right)\left(a_{4} b_{1} b_{5} a_{5} a_{3}\right)\left(b_{2} b_{4} c_{1} c_{4} c_{3} c_{2} c_{6}\right)\left(c_{5} a_{6}\right)\left(b_{3}\right)\left(b_{6}\right) .
\end{array}
$$

Then

$$
\begin{aligned}
& \sigma=\left(a_{4} b_{5} a_{5}\right)\left(b_{4} c_{4} c_{6}\right)\left(c_{5} c_{6}\right)\left(b_{6}\right), \\
& \pi_{1}=\left(a_{1} a_{2}\right), \quad a_{3} \rightarrow a_{4}, \\
& \pi_{2}=\left(b_{3}\right), \quad b_{1} \rightarrow b_{5}, \quad b_{2} \rightarrow b_{4}, \\
& \pi_{3}=c_{1} \rightarrow c_{4}, \quad c_{3} \rightarrow c_{2} \rightarrow c_{6} .
\end{aligned}
$$


3. The $\boldsymbol{\beta}$-extension of the MacMahon Master Theorem. Let $V_{m}$ be the determinant $\operatorname{det}\left(\delta_{i j}-b(i, j) x_{j}\right)(1 \leqq i, j \leqq m)$. The MacMahon Master Theorem asserts that the coefficient of $x_{1}^{n_{1}} \cdots x_{m}^{n_{m}}$ in the expansion of $V_{m}^{-1}$ is equal to the coefficient of $x_{1}^{n_{1}} \cdots x_{m}^{n_{m}}$ in the product

$$
\left(b(1,1) x_{1}+\cdots+b(1, m) x_{m}\right)^{n_{1}} \cdots\left(b(m, 1) x_{1}+\cdots+b(m, m) x_{m}\right)^{n_{m}} .
$$

It will be convenient to restate this statement in a slightly different form. Let $\mathscr{R}(\mathbf{n})$ denote the set of all the rearrangements

$$
r=r(1,1) \cdots r\left(1, n_{1}\right) \cdots r(m, 1) \cdots r\left(m, n_{m}\right)
$$

of the word $1^{n_{1}} \cdots m^{n_{m}}$ and let

$$
v(r)=\prod_{i, j} b(i, r(i, j)) \quad\left(1 \leqq i \leqq m ; 1 \leqq j \leqq n_{i}\right) .
$$

Clearly, the coefficient of $x_{1}^{n_{1}} \cdots x_{m}^{n_{m}}$ in (3.1) is equal to the sum of all the $v(r)$ with $r$ running over all the rearrangements of $1^{n_{1}} \cdots m^{n_{m}}$.

Next, consider a permutation $\pi$ belonging to $\mathscr{P}(\mathbf{n})$ (defined in $\S 1$ ). If $\pi$ sends $(i, j)$ over $\left(i^{\prime}, j^{\prime}\right)$, write $i^{\prime}=c \pi(i, j)$. Furthermore, define

$$
v(\pi)=\prod_{i, j} b(i, c \pi(i, j)) \quad\left(1 \leqq i \leqq m ; 1 \leqq j \leqq n_{i}\right) .
$$

For instance, if

$$
\pi=\left(\begin{array}{llllll}
a_{1,1} & a_{1,2} & a_{2,1} & a_{2,2} & a_{2,3} & a_{3,1} \\
a_{2,2} & a_{3,1} & a_{2,3} & a_{2,1} & a_{1,2} & a_{1,2}
\end{array}\right)
$$

belongs to $\mathscr{P}(2,3,1)$, then, $v(\pi)=b(1,2) b(1,3) b(2,2) b(2,2) b(2,1) b(3,1)$.

To each rearrangement $r$ in $\mathscr{R}(\mathbf{n})$ there correspond exactly $n_{1} ! \cdots n_{m}$ ! permutations $\pi$ in $\mathscr{P}(\mathbf{n})$ with the property that $v(\pi)=v(r)$. Therefore, the coefficient of $x_{1}^{n_{1}} \cdots x_{m}^{n_{m}}$ in (3.1) is also equal to

$$
\frac{1}{n_{1} ! \cdots n_{m} !} \sum_{\pi \in \mathscr{P}(\mathbf{n})} v(\pi)\left(=\frac{1}{n_{1} ! \cdots n_{m} !} v(\mathscr{P}(\mathbf{n}))\right) .
$$

The MacMahon Master identity can then be restated as

$$
\sum \frac{x_{1}^{n_{1}}}{n_{1} !} \cdots \frac{x_{m}^{n_{m}}}{n_{m} !} v(\mathscr{P}(\mathbf{n}))=V_{m}^{-1} .
$$

Next define the $\beta$-weight $v(\beta ; \pi)$ of each permutation $\pi$ in $\mathscr{P}(\mathbf{n})$ by

$$
v(\beta ; \pi)=\beta^{\mathrm{cyc} \pi} v(\pi) .
$$

THEOREM ( $\beta$-extension of the MacMahon Master Theorem). The following identity holds:

$$
\sum \frac{x_{1}^{n_{1}}}{n_{1} !} \cdots \frac{x_{m}^{n_{m}}}{n_{m} !} v(\beta ; \mathscr{P}(\mathbf{n}))=V_{m}^{-\beta} .
$$

Proof. Consider the partitional complex (see, e.g., [Fo], [Fo-Sch]) of the permutations and denote by $\mathscr{C} \mathscr{P}(\mathbf{n})$ the subset of the connected permutations in $\mathscr{P}(\mathbf{n})$. As the weight $v(\beta ; \cdot)$ is multiplicative [ Fo], [ Fo-Sch ], the following identity holds:

$$
\sum \frac{x_{1}^{n_{1}}}{n_{1} !} \cdots \frac{x_{m}^{n_{m}}}{n_{m} !} v(\beta ; \mathscr{P}(\mathbf{n}))=\exp \sum \frac{x_{1}^{n_{1}}}{n_{1} !} \cdots \frac{x_{m}^{n_{m}}}{n_{m} !} v(\beta ; \mathscr{C} \mathscr{P}(\mathbf{n}))
$$


From (3.3) and (3.6) with $\beta=1$

$$
\sum \frac{x_{1}^{n_{1}}}{n_{1} !} \cdots \frac{x_{m}^{n_{m}}}{n_{m} !} v(\mathscr{C} \mathscr{P}(\mathbf{n}))=-\log V_{m}
$$

But $v(\beta ; \pi)=\beta v(\pi)$ for every connected permutation, so that

$$
\sum \frac{x_{1}^{n_{1}}}{n_{1} !} \cdots \frac{x_{m}^{n_{m}}}{n_{m} !} v(\beta ; \mathscr{C} \mathscr{P}(\mathbf{n}))=-\beta \log V_{m},
$$

and finally (3.5) holds because of (3.6).

4. A combinatorial proof of Theorem 2. By the definition of the Laguerre polynomials

$$
n_{i} ! L_{n_{i}}^{(\alpha)}(x)=\sum_{k_{i}=0}^{n_{i}}(-1)^{k_{i}}\left(\begin{array}{l}
n_{i} \\
k_{i}
\end{array}\right)\left(\alpha+1+k_{i}\right)_{n_{i}-k_{i}} x^{k_{i}} \quad(i=1, \cdots, m) .
$$

In (1.3) and (1.5) expand each Laguerre polynomial and integrate term by term using the fact that $(1 / \Gamma(\alpha+1)) \int_{0}^{\infty} e^{-x} x^{n+\alpha} d x=(\alpha+1)_{n}$. This leads to:

$$
J(\mathbf{n} ; \Lambda ; \alpha)=\sum_{\mathbf{k}}(\alpha+1)_{k_{1}+\cdots+k_{m}} \prod_{i=1}^{m}\left(-\lambda_{i}\right)^{k_{i}}\left(\begin{array}{c}
n_{i} \\
k_{i}
\end{array}\right)\left(\alpha+1+k_{i}\right)_{n_{i}-k_{i}},
$$

where the first summation is over all sequences $\mathbf{k}=\left(k_{1}, \cdots, k_{m}\right)$ with $0 \leqq k_{1} \leqq n_{1}, \cdots$, $0 \leqq k_{m} \leqq n_{m}$. From the lemma of $\S 2, J(\mathbf{n} ; \Lambda ; \alpha)$ can also be written as

$$
J(\mathbf{n} ; \Lambda ; \alpha)=\sum_{\mathbf{T}} \prod_{i=1}^{m}\left(-\lambda_{i}\right)^{\left|A_{i} \backslash T_{i}\right|} \sum_{\pi} w(\pi),
$$

where the first summation is over all sequences $\mathbf{T}=\left(T_{1}, \cdots, T_{m}\right)$ such that $T_{i} \subset A_{i}$ for all $i$ and the second one over all permutations $\pi$ satisfying $T_{i} \subset \operatorname{Inc}_{i} \pi$ for all $i$. Hence,

$$
\begin{aligned}
J(\mathbf{n} ; \Lambda ; \alpha) & =\sum_{\pi} w(\pi) \sum_{\mathbf{T}} \prod_{i=1}^{m}\left(-\lambda_{i}\right)^{\left|A_{i} \backslash T_{i}\right|} \quad\left[\text { with } T_{i} \subset \operatorname{Inc}_{i} \pi\right] \\
& =\sum_{\pi} w(\pi) \prod_{i=1}^{m}\left(-\lambda_{i}\right)^{\left|A_{i} \backslash \operatorname{Inc}_{i} \pi\right|} \sum_{\mathbf{T}} \prod_{i=1}^{m}\left(-\lambda_{i}\right)^{\left|\operatorname{Inc}_{i} \pi \backslash T_{i}\right|} \quad \text { [with } T_{i} \subset \operatorname{Inc}_{i} \pi \text { ] } \\
& =\sum_{\pi} w(\pi) \prod_{i=1}^{m}\left(-\lambda_{i}\right)^{\left|A_{i} \backslash \operatorname{Inc}_{i} \pi\right|} \prod_{i=1}^{m}\left(1-\lambda_{i}\right)^{\left|\operatorname{Inc}_{i} \pi\right|},
\end{aligned}
$$

the binomial identity being used in the last step of that derivation. Thus,

$$
J(\mathbf{n} ; \Lambda ; \alpha)=\sum_{\pi} W(\pi),
$$

which is exactly the statement of Theorem 2 .

5. An analytical proof of Theorem 2. There is another way of expressing the $W$ weight. For each ordered pair $(i, j)$ of integers lying between one and $m$ and each permutation $\pi$ in $\mathscr{P}(\mathbf{n})$ let $\gamma_{i j}=\gamma_{i j}(\pi)$ be the number of elements $x$ in $A_{i}$ that are mapped into $A_{j}$ (i.e., $\gamma_{i j}=\left|\pi\left(A_{i}\right) \cap A_{j}\right|$ ). Define the $W^{\prime}$-weight of $\pi$ by

$$
W^{\prime}(\pi)=w(\pi) \prod_{i=1}^{m}\left(1-\lambda_{i}\right)^{\gamma_{i i}} \prod_{\substack{i, j \\ i \neq j}}\left(-\sqrt{\lambda_{i} \lambda_{j}}\right)^{\gamma_{i j}}
$$


LEMMA. We have $W^{\prime}(\pi)=W(\pi)$.

Proof. As $\sum_{i, j ; i \neq j} \gamma_{i j}=\sum_{i}\left|\operatorname{Inc}_{i} \pi\right|$, it suffices to show that

$$
\prod_{i} \lambda_{i}^{\left|A_{i} \backslash \operatorname{Inc}_{i} \pi\right|}=\prod_{\substack{i, j \\ i \neq j}}\left(\sqrt{\lambda_{i} \lambda_{j}}\right)^{\gamma_{i j}} .
$$

For each $x \in A$ let $c(x)=i$, if and only if $x \in A_{i}$. Then

$$
\prod_{i} \lambda_{i}^{\left|A_{i} \backslash \operatorname{Inc}_{i} \pi\right|}=\prod_{x} \lambda_{c(x)} \quad(x \in A \backslash \operatorname{Inc} \pi) .
$$

For each nonincestuous element $x$ let $x^{*}$ be the nearest nonincestuous element to $x$ in the following sense. Let $k=\min \left\{l: l \geqq 1, \pi^{l}(x) \in A \backslash \operatorname{Inc} \pi\right\}$ and put $x^{*}=\pi^{k}(x)$. Clearly, $x^{*}$ is uniquely defined and $x \rightarrow x^{*}$ is a bijection of $A \backslash$ Inc $\pi$ onto itself. Then

$$
\prod_{x} \lambda_{c(x)}=\prod_{x} \sqrt{\lambda_{c(x)} \lambda_{c\left(x^{*}\right)}} \quad(x \in A \backslash \operatorname{Inc} \pi) .
$$

As $c\left(x^{*}\right)=c(\pi(x))$, we also have

$$
\prod_{x} \lambda_{c(x)}=\prod_{x} \sqrt{\lambda_{c(x)} \lambda_{c(\pi(x))}}=\prod_{\substack{i, j \\ i \neq j}}\left(\sqrt{\lambda_{i} \lambda_{j}}\right)^{\gamma_{i j}} .
$$

In the notation of $\S 3$ take

$$
b(i, j)=\left\{\begin{array}{lll}
1-\lambda_{i} & \text { if } & i=j, \\
-\sqrt{\lambda_{i} \lambda_{j}} & \text { if } & i \neq j .
\end{array}\right.
$$

The determinant $V_{m}=\operatorname{det}\left(\delta_{i j}-b(i, j) x_{j}\right)$ has been calculated by Askey et al. [ As-IsKo, Thm. 4]. They showed that a simple induction argument gives

$$
V_{m}=\prod_{i=1}^{m}\left(1-x_{i}\right)+\sum_{i=1}^{m} \lambda_{i} x_{i} \prod_{j \neq i}\left(1-x_{j}\right) .
$$

On the other hand, the generating function (1.1) for the Laguerre polynomials yields the identity

$$
\sum_{\mathbf{n}} J(\mathbf{n} ; \Lambda ; \alpha) \frac{x^{n_{1}}}{n_{1} !} \cdots \frac{x^{n_{m}}}{n_{m} !}=V_{m}^{-(\alpha+1)},
$$

almost immediately, as noticed by the same authors [ As-Is-Ko, Thm. 2]. By comparing (5.4) with (3.5), we conclude that

$$
J(\mathbf{n} ; \Lambda ; \alpha)=v(\alpha+1 ; \mathscr{P}(\mathbf{n})) .
$$

Finally, expand $v(\alpha+1 ; \pi)$ in terms of the $b(i, j)$ 's:

$$
v(\alpha+1 ; \pi)=(\alpha+1)^{\mathrm{cyc} \pi} \prod_{i, j} b(i, c \pi(i, j))=W^{\prime}(\pi) .
$$

Then, Theorem 2 is proved by using the previous lemma.

6. Positivity. As mentioned in the Introduction, the positivity of $J(n ; \Lambda ; \alpha)$ for $m=3$ (Koornwinder's inequality) implies a general result for an arbitrary $m$ by means of the orthogonality relation and the old expansion of the Laguerre polynomials [ As-IsKo]. However, that inequality remains the crucial point. It is next restated and re-proved.

THEOREM 3. Let $m=3$ and $\Lambda=\left(\lambda_{1}=\lambda, \lambda_{2}=1-\lambda, \lambda_{3}=1\right)$. Then, for $\alpha \geqq 0$ and $0 \leqq \lambda \leqq 1$ we have $J(\mathbf{n}, \Lambda ; \alpha) \geqq 0$. 
Proof. For $m=3$ the summation $\Sigma_{\pi} W(\pi)$ in (1.7) becomes

$$
\sum_{\pi} W(\pi)=\sum_{\pi}(\alpha+1)^{\mathrm{cyc} \pi} \prod_{i=1}^{3}\left(1-\lambda_{i}\right)^{\left|\operatorname{Inc}_{i} \pi\right|}\left(-\lambda_{i}\right)^{\left|A_{i} \backslash \operatorname{Inc}_{i} \pi\right|},
$$

where the summation is over the permutations of $\pi$ of $A=A_{1}+A_{2}+A_{3}$ having no incestuous elements in $A_{3}$ (because $\lambda_{3}=1$ ). As in $\S 5$ let $\gamma_{i j}=\left|\pi\left(A_{i}\right) \cap A_{j}\right|$ be the number of elements that go from $A_{i}$ to $A_{j}$. The sign of the $W$-weight of $\pi$ is equal to $(-1)^{s(\pi)}$, where

$$
s(\pi)=\gamma_{12}+\gamma_{13}+\gamma_{21}+\gamma_{23}+\gamma_{31}+\gamma_{32}=2\left(n_{1}+n_{3}-\gamma_{11}\right)-\left(\gamma_{13}+\gamma_{31}\right) .
$$

Thus

$$
\operatorname{sgn} W(\pi)=(-1)^{\gamma_{13}+\gamma_{31}} .
$$

Furthermore, the exponent of $\lambda_{1}$ in $W(\pi)$ is equal to

$$
\gamma_{12}+\gamma_{13}+\gamma_{22}=n_{2}-n_{3}+\gamma_{13}+\gamma_{31}
$$

while the exponent of $\lambda_{2}$ is equal to $\gamma_{11}+\gamma_{21}+\gamma_{23}=n_{1}+n_{3}-\gamma_{13}-\gamma_{31}$. Hence

$$
W(\pi)=(\alpha+1)^{\mathrm{cyc} \pi}(-1)^{\gamma_{13}+\gamma_{31}} \lambda_{1}^{n_{1}-n_{3}+\gamma_{13}+\gamma_{31}} \lambda_{2}^{n_{1}+n_{3}-\gamma_{13}-\gamma_{31}} .
$$

Thus, when $\alpha=0$, the weight $W(\pi)$ depends only on $\gamma_{13}$ an $\gamma_{31}$. Assume that $\alpha=0$ and compute the number of permutations $\pi$ such that $\gamma_{13}=a$ and $\gamma_{31}=b$. This is easy if we have in mind the representation of each permutation as a two-line matrix. The number of ways of placing the elements of the set $\pi\left(A_{1}\right) \cap A_{3}$ and $\pi\left(A_{2}\right) \cap A_{3}$ is equal to $\left(\begin{array}{c}n_{1} \\ a\end{array}\right)\left(\begin{array}{c}n_{3} \\ n_{3}-a\end{array}\right) n_{3}$ !. In the same manner, $\left(\begin{array}{c}n_{1} \\ b\end{array}\right)\left(\begin{array}{c}n_{3} \\ n_{3}-b\end{array}\right) n_{3}$ ! is the number of ways of placing the elements of $\pi\left(A_{3}\right) \cap A_{1}$ and $\pi\left(A_{3}\right) \cap A_{2}$. There remain $\left(n_{1}+n_{2}-n_{3}\right)$ ! positions for the other elements to be placed. Thus,

$$
\begin{aligned}
\sum_{\pi} W(\pi)= & \sum_{a, b}(-1)^{a+b}\left(\begin{array}{c}
n_{1} \\
a
\end{array}\right)\left(\begin{array}{c}
n_{2} \\
n_{3}-a
\end{array}\right) n_{3} !\left(\begin{array}{c}
n_{1} \\
b
\end{array}\right)\left(\begin{array}{c}
n_{2} \\
n_{3}-b
\end{array}\right) n_{3} ! \\
& \times\left(n_{1}+n_{2}-n_{3}\right) ! \lambda_{1}^{n_{1}-n_{3}+a+b} \lambda_{2}^{n_{1}+n_{3}-a-b} \\
= & \left(n_{3} !\right)^{2} \lambda^{n_{2}-n_{3}} \lambda_{2}^{n_{1}+n_{3}}\left(n_{1}+n_{2}-n_{3}\right) ! \\
& \times\left(\sum_{a}\left(\begin{array}{c}
n_{1} \\
a
\end{array}\right)\left(\begin{array}{c}
n_{2} \\
n_{3}-a
\end{array}\right)(-1)^{a}\left(\frac{\lambda_{1}}{\lambda_{2}}\right)^{a}\right)^{2} \\
\geqq & 0 .
\end{aligned}
$$

This proves Koornwinder's inequality for $\alpha=0$.

For an arbitrary $\alpha>0$ let us make use again of the argument of Gillis, Reznick, and Zeilberger [ Gi-Re-Ze, Prop. 1]. The determinant $V_{3}$ corresponding to the previous $W$ is easy to calculate, either directly, or by means of $(5.3)$ :

$$
V_{3}=1-\lambda_{2} x_{1}-\lambda_{1} x_{2}-x_{3}\left[\lambda_{1} x_{1}+\lambda_{2} x_{2}-x_{1} x_{2}\right] \text {. }
$$

Put $X=1-\lambda_{2} x_{1}-\lambda_{1} x_{2}$ and $Y=\lambda_{1} x_{1}+\lambda_{2} x_{2}-x_{1} x_{2}$, so that $V_{3}=X-x_{3} Y$. Hence

$$
\begin{aligned}
V_{3}^{-(\alpha+1)} & =X^{-(\alpha+1)}\left[1-x_{3} Y X^{-1}\right]^{-(\alpha+1)} \\
& =X^{-\alpha} \sum_{r} \frac{(\alpha+1)_{r}}{r !} \frac{Y^{r}}{X^{r+1}} x_{3}^{r} .
\end{aligned}
$$


But $V_{3}^{-1}=\left(X-x_{3} Y\right)^{-1}=\sum_{r}\left(Y^{r} / X^{r+1}\right) x_{3}^{r}$ has positive coefficients. This is another way of saying that Koornwinder's inequality holds for $\alpha=0$. Hence the same inequality is true for every $\alpha \geqq 0$.

Remark. The idea of expressing the summation $\Sigma_{\pi} W(\pi)$ for $\alpha=0$ as a square of a polynomial is basically due to Ismail and Tamhankar [ Is-Ta], even though they made their calculations with the determinant $V_{3}$ itself. The proof given above is only an adaptation of the derivation of Gillis and Zeilberger [ $\mathrm{Gi}-\mathrm{Ze}$ ] to the permutation combinatorial set-up developed in this paper.

Acknowledgment. The first author thanks Jiang Zeng for bringing his attention to the fact that Theorem 1 is just a particular case of Theorem 2 .

\section{REFERENCES}

[ As1] R. ASKEY, Linearization of the product of orthogonal polynomials, in Problems in Analysis (A symposium in honor of Salomon Bochner), Robert C. Gunning, ed., Princeton University Press, Princeton, NJ, 1970, pp. 131-138.

[ As2] - Orthogonal and Special Functions, CBMS-NSF Regional Conference Series in Applied Mathematics 21, Society for Industrial and Applied Mathematics, Philadelphia, PA, 1975.

[ As-Is] R. ASKEY AND M. E. H. IsmaIL, Permutation problems and special functions, Canad. J. Math. 28 (1976), pp. 853-874.

[ As-Is-Ko] R. ASKEY, M. E. H. ISMAIL, AND T. KOORNWINDER, Weighted permutation problems and Laguerre polynomials, J. Combin. Theory Ser. A, 25 (1978), pp. 277-287.

[As-Ga] R. ASKEY AND G. GASPER, Convolution structures for Laguerre polynomials, J. d'Anal. Math., 31 (1977), pp. 46-48.

[ Ev-Gi] S. Even AND J. Gillis, Derangements and Laguerre polynomials, Proc. Cambridge Phil. Soc., 79 (1976), pp. 135-143.

[ Fo] D. FOATA, La périe génératrice exponentielle dans les problèmes d'énumération, Presses de l'Université de Montréal, Montréal, Quebec, Canada, 1974.

[ Fo-Sch] D. FOATA AND M.-P. SCHUTZENBERGER, Théorie géométrique des polynômes eutériens, Lecture Notes in Mathematics 138, Springer-Verlag, Berlin, 1970.

[ Fo-St ] D. FOATA AND V. STREHL, Combinatorics of Laguerre polynomials, in Enumeration and Design, D. M. Jackson and S. A. Vanstone, eds., Waterloo, Ontario, Canada, June-July 1982, pp. 123140; Academic Press, Toronto, 1984.

[Gi-Ze] J. Gillis AND D. ZeILBERGER, $A$ direct combinatorial proof of a positivity result, European J. Combin., 4 (1983), pp. 221-223.

[Gi-Re-Ze] J. Gillis, B. REZNICK, AND D. ZEILBERGER, On elementary methods in positivity theory, SIAM J. Math. Anal., 14 (1983), pp. 396-398.

[ Is-Ta] M. E. H. ISMAIL AND M. V. TAMHANKAR, A combinatorial approach to some positivity problems, SIAM J. Math. Anal., 10 (1979), pp. 478-485.

[ Ja] D. M. JACKSON, Laguerre polynomials and derangements, Proc. Cambridge Phil. Soc., 80 (1976), pp. 213-214.

[ Ko] T. KOORNwINDER, Positivity proofs for linearization and connection coefficients of orthogonal polynomials satisfying an addition theorem, J. London Math. Soc., 18 (1978), pp. 101-114.

[ Mac] Percy AleXander MacMahon, Combinatory Analysis, Vol. 1, Cambridge, University Press, 1915; reprinted by Chelsea, New York, 1955.

[ Ra] M. RAHMAN, A non-negative representation of the linearization coefficients of the product of Jacobi polynomials, Canad. J. Math., 33 (1981), pp. 915-928.

[ Ri] J. RioRdan, An Introduction to Combinatorial Analysis, New York, John Wiley, 1959.

[ Sa-Vi] M. DE SAINTE-CATHERINE AND G. VIENNOT, Combinatorial interpretation of integrals of products of Hermite, Laguerre and Tchebycheff polynomials, in Polynômes orthogonaux et applications, C. Brezinski, et al. eds., Bar-le-Duc, 1984, pp. 120-128; Lecture Notes in Mathematics 1175, Springer-Verlag, Berlin, 1985.

[ Ze] J. ZENG, Linéarisation de produits de polynômes de Meixner, Krawtchouk et Charlier, Strasbourg, 1987, submitted. 